\title{
Discrete Cosine Transformation for image compression: A comparative study
}

\author{
Chong Zeng ${ }^{a}$, Jiali Chen ${ }^{b}$ and Xiaojie Chen ${ }^{c}$ \\ Longyan University, Longyan 364000, China. \\ azengchong111@163.com, bchenjialicj|425@163.com, 'chxj856@163.com
}

Keywords: Compression (100.0100), DCT (100.0100), JPEG (100.0100).

Abstract. In this paper, an experimental method has been proposed to determine the Discrete Cosine Transformation how to work on the JPEG image compression and compare the computational complexity on different forms of DCT, which include common 2D-DCT, fast 2D-DCT (FFDCT and DFDCT) and matrix DCT method.

\section{Introduction}

DCT is the most common image compression technique applied in different standard compression algorithms such as Motion Pictures Expert Group (MPEG) standard and H.261, H.264 [20]. DCT was defined by [2], which can be computed by the discrete Fourier transform (DFT) adopt fast Fourier transform (FFT) algorithm [1]. And the DCT is used to transform a signal from time or spatial domain to the frequency domain. In digital image compression, the DCT is converting an image pixel pattern into a set of spatial frequency values, which attempts to reduce the correlation between images data.

In order to understand the performance of DCT in depth, some basic measurements will be adopted to measure the discrete cosine transform (DCT). Such as compression efficiency, Complexity, and distortion measurement (DCT is a lossy algorithm). I will adopts four measurements. The entropy of image, Root-Mean-Square Error, and compression ratio are chosen to measure the property of DCT. The computational complexity and operation runtime are used to compare the difference DCT method.

\section{Experiment}

(a)original image

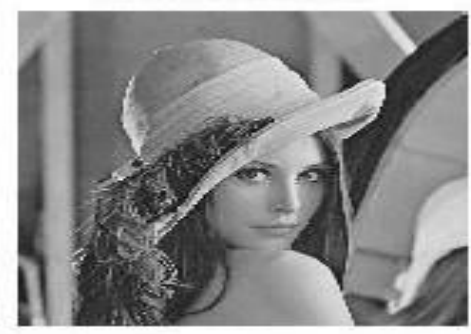

(c)reconstructed image

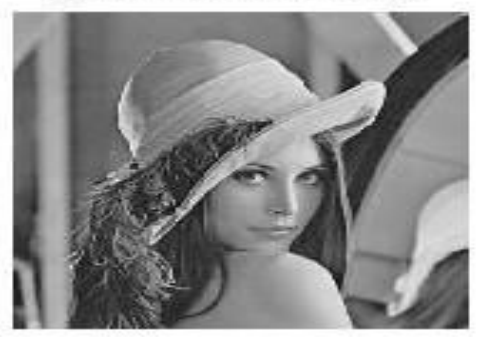

(b)transformed image

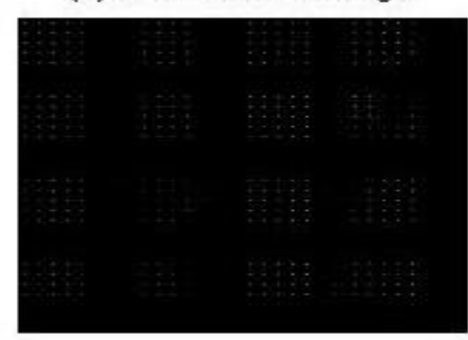

(d)redundancy image

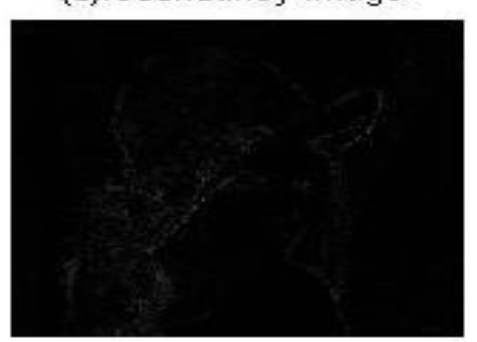

Fig.1(a) original image, (b) transformed image, (c) reconstructed image, (d) redundancy image 
Matlab simulation experiment will help us to understand how the JPEG and DCT working and what the difference between each DCT method. The experiment chooses gray scale image, which using luminance level to represent each pixel [3]. And two still image formats were chosen, one is bmp format which used mainly for simple uncompressed images, and the other is tiff format which support a variety of image compression standards [4].The experimental result as follows:

The first experiment is picked the JPEG method to compress lena image (see JPEG_Mask.m). So we could get the transformed image, reconstructed image and the redundancy image which obtained by using original image minus reconstructed image.

From Figure 1, under the human eyes obviation, there is no difference between original image and reconstructed image. And the redundancy image clearly shows that some image information was compressed by DCT operation.

The experiment two is Adopt the JPEG methods to compress lena image.

Table 1 Original matrix

\begin{tabular}{|c|c|c|c|c|c|c|c|}
\hline 2.7402 & -0.2565 & -0.076717 & -0.023465 & 0.017647 & 0.0081819 & 0.0094926 & 0.0017205 \\
\hline-0.33081 & -0.07205 & 0.0028341 & -0.0124 & 0.012176 & -0.01181 & -0.005645 & 0.020915 \\
\hline-0.036655 & 0.0068295 & 0.053066 & -0.016529 & 0.034677 & 0.028427 & 0.0050353 & -0.0086337 \\
\hline-0.034742 & 0.0068693 & 0.0032753 & 0.0043378 & 0.002492 & 0.020042 & 0.0097679 & -0.0056695 \\
\hline-0.026471 & 0.022689 & 0.0068709 & -0.0011498 & -0.0039216 & 0.010946 & -0.003907 & -0.012415 \\
\hline-0.016558 & 0.017623 & 0.007977 & -0.0036472 & 0.0049163 & 0.0025861 & -0.003090 & -0.0091738 \\
\hline-0.034692 & -0.013349 & -0.017514 & 0.010531 & 0.0068599 & -0.016797 & 0.0086986 & 0.0089101 \\
\hline 0.010523 & -0.0066958 & -0.00064949 & 0.0082454 & 0.01366 & -0.018471 & 0.0055 & 0.0082633 \\
\hline
\end{tabular}

Table 2 Quantized matrix

\begin{tabular}{|c|c|c|c|c|c|c|c|}
\hline 0.17126 & -0.023319 & -0.076717 & -0.0014666 & 0.0007352 & 0.0002045 & 0.0001861 & $2.8205 \mathrm{e}-00$ \\
\hline-0.027567 & -0.0060042 & 0.00020243 & -0.0006526 & 0.0004683 & -0.000203 & -9.4099 & 0.00038027 \\
\hline-0.002618 & 0.00052534 & 0.0033166 & -0.0006887 & 0.0008669 & 0.0004987 & $7.2976 \mathrm{e}-0$ & -0.0001541 \\
\hline-0.002481 & 0.00040408 & 0.00014888 & 0.00014958 & 0.002492 & 0.020042 & 0.0097679 & -0.0056695 \\
\hline-0.001470 & 0.0010313 & 0.0001857 & $-2.0531 \mathrm{e}-00$ & $-5.767 \mathrm{e}-00$ & 0.0001004 & $-3.7934 \mathrm{e}-$ & -0.0001612 \\
\hline-0.000689 & 0.00050351 & 0.00014504 & $-5.6987 \mathrm{e}-00$ & $6.0696 \mathrm{e}-00$ & $2.4867 \mathrm{e}-0$ & $-2.735 \mathrm{e}-0$ & $-9.9715 \mathrm{e}-00$ \\
\hline-0.000708 & -0.0002085 & -0.00022453 & 0.00012104 & $6.6601 \mathrm{e}-00$ & -0.000138 & $7.2488 \mathrm{e}-0$ & $8.8219 \mathrm{e}-00$ \\
\hline 0.0001461 & $-7.278 \mathrm{e}-005$ & $-6.8367 \mathrm{e}-00$ & $8.4137 \mathrm{e}-00$ & 0.0001219 & -0.000184 & $5.3398 \mathrm{e}-0$ & $8.3467 \mathrm{e}-00$ \\
\hline
\end{tabular}

From above table, the original matrix is the pixel value of one subimage. The quantized matrix can be obtained by quantized original matrix, and the value on the top left corner of matrix become smaller. The reason is that the quantization matrix Table.2. is generated according to heuristically determined perceptual or visual psych importance [5]. Overall, the image can be compressed by using quantization matrix and zonal matrix.

The experiment three is adopting JPEG method on three different images.

Table 3 Erms and compression ratio

\begin{tabular}{|c|c|c|}
\hline Test image & Erms (bits) & Compression ratio \\
\hline lena(512*512) & 0.0252 & 4.8817 \\
\hline trui $(256 * 256)$ & 0.0137 & 5.4196 \\
\hline photo $(128 * 128)$ & 0.0528 & 5.3980 \\
\hline
\end{tabular}

From the Table.3, the Erms value always tiny, but compression ratio is big. It means that we could obtain a good quality of restored image with high compressed through adopt JPEG method. The bigger ratio represents that more redundancy of image are removed.

The experiment four is adopt JPEG method but using different retained coefficients method to compress lena.jpg. If only 1,10 or 25 coefficients be retained, then the corresponding restored image could show as Figure2. It clearly found that retain more coefficients could get higher quality image. 


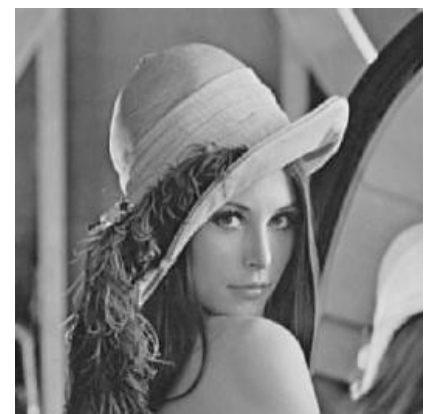

(a)

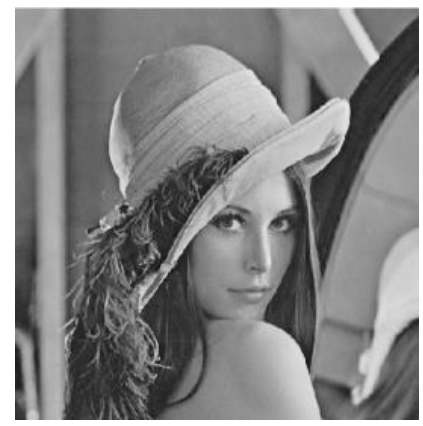

(c)

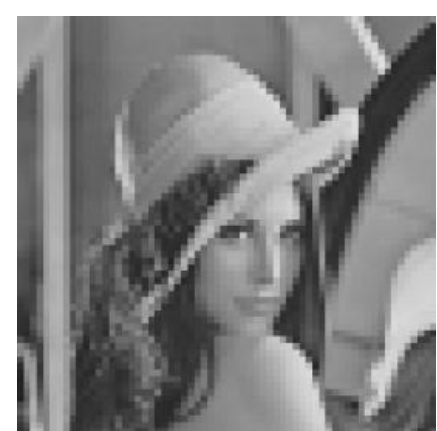

(b)

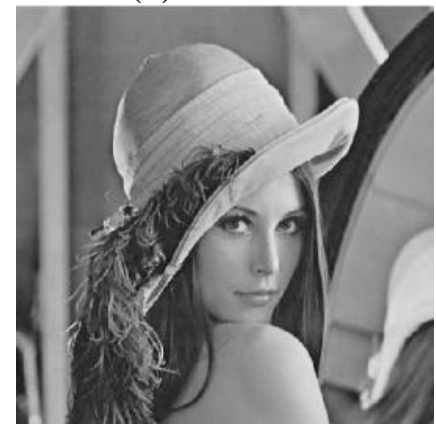

(d)

Fig. 2 (a) Original image (b) Retain 1 coefficients (c) Retain 10 coefficient (d) Retain 25 coefficients

\section{Conclusion}

In this paper, we adopts information theory measurements to investigate the performance of DCT. It can be found the optimum block size of DCT is $8 * 8$ or $16^{*} 16$, which through comparing RootMean-Square Error (Erms) of different block size of image. Furthermore, after comparing the entropy reduction of different image, we can prove that DCT could reduce the image information and it cannot gain perfect quality of reconstructed image after losing certain frequencies. The bigger value of compression ratio can be get after adopt DCT, that is, the DCT provides perfect compression effect. And then, by compare compression ratio to its Erms, the higher quality of reconstructed image results in lower compression ratio. As the DCT is more efficient and practical in image compression procession, JPEG and MPEG standards are select it.

\section{Acknowledgments}

The authors would like to thank Prof. Wallace Andrew of Heriot-Watt University for his helpful discussion. This work is partially supported by important program of LongYan City under the contract number 2014LY10 and LQ2013006.

\section{References}

[1] X. Chen, R. Eifrig, A. Luthra, K. Panusopone, Coding of an arbitrary shaped interlaced video in MPEG-4, in: Proceedings of the IEEE International Conference on Acoustics, Speech and Signal Processing (ICASSP'99), 1999 pp. 3121-3124.

[2] N. Ahmed, T. Natarajan, and K. R. Rao, Discrete Cosine Transform, IEEE. Trans. Computer, Vol. C-23, pp. 90-93, Jan 1974.

[3] Weidong Kou, "DIGITAL IMAGE COMPRESSION Algorithms and Standards", 1995, ISBN 0-7923-9626-X.

[4] Rafael C.Gonzalez, Richard E.Woods, "Digital Image Processing (Third Edition)", pp.525-623.

[5] Rafael C.Gonzalez, Richard E.Woods, "Digital Image Processing (second Edition)", pp.409-514. 